\title{
Investigations for the model-based dynamic calibration of force transducers by using shock excitation
}

\author{
Michael Kobusch ${ }^{1}$, Sascha Eichstädt ${ }^{2}$, Leonard Klaus ${ }^{1}$, Thomas Bruns ${ }^{1}$ \\ ${ }^{1}$ Physikalisch-Technische Bundesanstalt (PTB), Bundesallee 100, 38116 Braunschweig, Germany \\ ${ }^{2}$ Physikalisch-Technische Bundesanstalt (PTB), Abbestr. 2-12, 10587 Berlin, Germany
}

\begin{abstract}
Within the scope of the joint research project EMRP IND09 "Traceable dynamic measurements of mechanical quantities", numerous measurements were performed at PTB's $20 \mathrm{kN}$ primary shock force calibration device to investigate and validate the approach of a model-based dynamic calibration of force transducers by using shock excitations. The tests included several strain gauge force transducers of greatly differing structural design, size, weight and mechanical coupling. By looking at a few examples, some investigated physical models of the measurement set-up and a developed data analysis procedure for parameter identification based on measured shock data are presented and discussed. The models reproduce the dynamic response including the observed modal oscillations of various origins that limit the usable measurement bandwidth. Moreover, these modal oscillations may have an important role for the parameter identification process, which is further discussed. This paper is an extended version of the original contribution to the IMEKO 2014 conference in Cape Town, South Africa.
\end{abstract}

\section{Section: RESEARCH PAPER}

Keywords: Dynamic calibration; shock force; dynamic modelling; parameter identification

Citation: Michael Kobusch, Sascha Eichstädt, Leonard Klaus, Thomas Bruns, Investigations for the model-based dynamic calibration of force transducers by using shock excitation, Acta IMEKO, vol. 4, no. 2, article 8, June 2015, identifier: IMEKO-ACTA-04 (2015)-02-08

Editor: Paolo Carbone, University of Perugia, Italy

Received September 29, 2014; In final form February 13, 2015; Published June 2015

Copyright: (ㄷ 2015 IMEKO. This is an open-access article distributed under the terms of the Creative Commons Attribution 3.0 License, which permits unrestricted use, distribution, and reproduction in any medium, provided the original author and source are credited.

Funding: This work is part of the Joint Research Project IND09 "Traceable dynamic measurement of mechanical quantities" of the European Metrology Research Programme (EMRP). The EMRP is jointly funded by the EMRP participating countries within EURAMET and the European Union.

Corresponding author: Michael Kobusch, e-mail: michael.kobusch@ptb.de

\section{INTRODUCTION}

Dynamic force measurements are widely used in many industrial areas, and the increasing demands on measurement accuracy have set new metrological challenges. But still, traceability for dynamic measurements is solely based on static calibrations, and documentary standards or commonly accepted guidelines for dynamic measurements do not exist. For this reason, the establishment of traceable measurements under dynamic conditions is a highly relevant topic. Its importance is emphasized by a current European Metrology Research Programme (EMRP) joint research project dedicated to the traceable dynamic measurement of mechanical quantities [1].

In this context, the general approach of a model-based calibration methodology will be followed in which the dynamic behaviour of the force transducer in a given mechanical calibration set-up is described by an appropriate model consisting of a series arrangement of spring-massdamper elements. The characteristic dynamic model parameters of the force transducer - i.e. values describing its distribution of mass, stiffness and damping - have to be determined. By fitting modelled and measured shock force data, the parameters of interest may be identified from the dynamic measurements. Considering the fact that the measurement data may show modal oscillations of the mechanical set-up of various sources, which were found in previous experimental studies supported by finite element simulations [2], the development and selection of adequate methods and procedures to analyse the shock force data is of great importance.

The general purpose of a model-based calibration is the determination of the characteristic parameters which define the force transducer's dynamic measurement behaviour in a given dynamic application. The parametric model of the transducer could in principle be employed as part of a larger parametric model of the particular measurement application, for instance of a shock calibration device or a uni-axial testing machine. The approach of the model-based calibration will furthermore allow the calculation of realistic measurement uncertainties in 


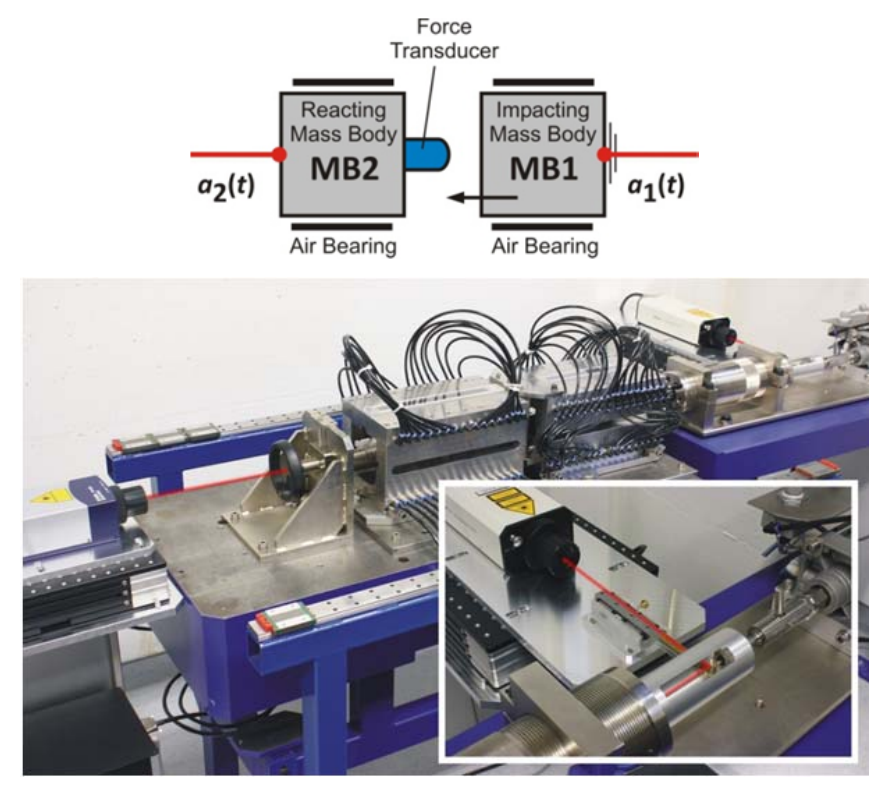

Figure 1. The $20 \mathrm{kN}$ shock force calibration device at PTB:

schematic diagram of the working principle (top), photographs (bottom) of the device with visualized beams of the laser interferometers, the inset shows the beam deflection at the spring-driven acceleration mechanism.

dynamic force measurements, which are basically not known at the moment.

The remainder of this paper is outlined as follows. Section 2 presents experimental results from shock force calibration tests performed with different force transducers. Section 3 then discusses the mathematical modelling and the parameter identification procedure. Finally, the last two sections give an outlook to future research and conclude the presented work.

\section{EXPERIMENTAL TESTS}

Within the scope of the above-mentioned EMRP project, several strain gauge force transducers of greatly differing size, weight, mechanical design and adaptation were investigated at PTB's $20 \mathrm{kN}$ primary shock force calibration device.

\subsection{Shock calibration device}

Figure 1 shows the calibration device and illustrates its working principle. Two cube-shaped mass bodies (MB1, MB2) each of $10 \mathrm{~kg}$ and the force transducer under test are brought to collision. The traceability of the shock force is realized by the determination of mass (from weighing) and acceleration (by means of laser vibrometers). Further information about this device is given in [3]. A recent modification of its measurement geometry now allows on-axis vibrometer measurements similar to those obtained with a larger $250 \mathrm{kN}$ calibration device [4]. As
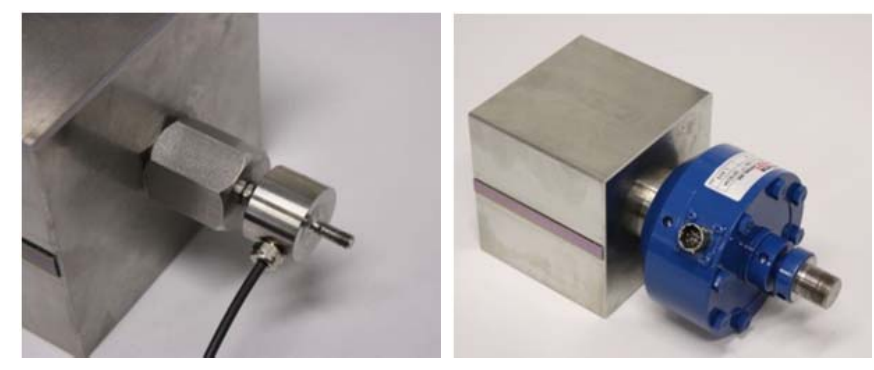

Figure 2. Mounted strain gauge force transducers: HBM U9B / 1 kN (left), Interface 1610 / 2.2 kN (right). this geometry is not susceptible to parasitic rotational vibrations possibly excited by the impact [5], the quality of the timedependent acceleration signals $a_{1}$ and $a_{2}$ has been improved.

\subsection{Force transducers}

The tested force transducers are equipped with a threaded bolt with a spherical end face, and can measure compression as well as tension forces. This choice also allows calibrations with sinusoidal forces for comparing the different dynamic results. Figure 2 shows two investigated force transducers and their adaptation to the mass body of the calibration device.

The small HBM U9B / $1 \mathrm{kN}$ has a mass of about $63 \mathrm{~g}$ and uses a measuring body with a flexing diaphragm applied with strain gauges that ends in the upper threaded bolt. This diaphragm, which is considered as the measuring spring, divides the mechanical structure of the force transducer into two parts. The effective mass of the upper part (head mass, see Section 3.1 ) is less than $3 \mathrm{~g}$. The manufacturer specifies a fundamental resonance of $24 \mathrm{kHz}$.

In contrast, the large Interface $1610 / 2.2 \mathrm{kN}$ is a shear beam strain gauge transducer of more than $1.5 \mathrm{~kg}$ (including the connector) and a diameter of $105 \mathrm{~mm}$. This transducer hardly fits into the squared air bearing of $108 \mathrm{~mm}$ clearance and thus clearly marks the maximum size for the $20 \mathrm{kN}$ shock force calibration device.

Former tests with an even larger force transducer of similar design (Interface $1032 / 225 \mathrm{kN}$ ) performed at the $250 \mathrm{kN}$ shock calibration device showed that the shock response can be predominately influenced by a comparably low coupling resonance [2], [6], which denotes the vibration of the transducer against its fixation on the reacting mass body. Regarding the small HBM U9B with its thin threaded bolt that connects to the base adapter, similar behaviour might also be expected.

\subsection{Shock force measurements}

Typical examples of measured shock force signals are shown in Figure 3. The small HBM U9B / $1 \mathrm{kN}$ measured a very smooth pulse without post-impact signal ringing. In contrast, the much heavier Interface $1610 / 2.2 \mathrm{kN}$ responded with a shorter pulse with superposed oscillations and prominent signal ringing. The plots demonstrate the possible variety of responses in the time domain. Different types of transducers with their specific mechanical adaptations can respond quite differently.

The tests used a hard metallic shock contact in order to achieve short pulses that are capable of exciting the transducer's mechanical resonances, i.e. no pulse-shaping material was applied between the impact surfaces. Under these conditions, the impacting mass body of $10 \mathrm{~kg}$ generated shock pulses of a duration of about one millisecond.
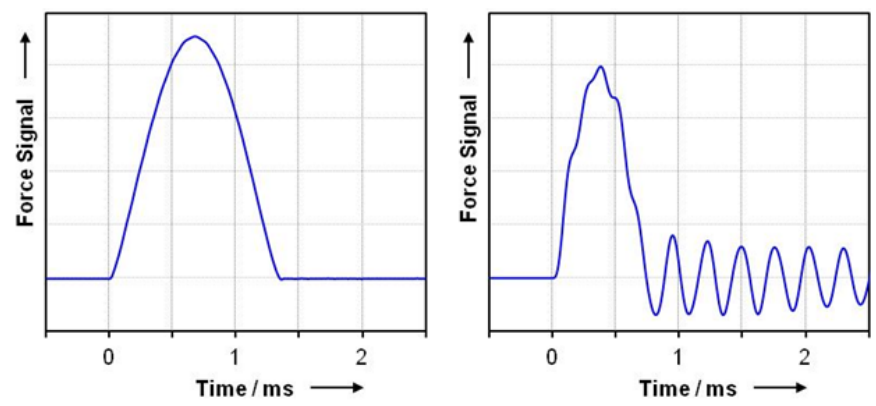

Figure 3. Measured shock force pulses: force transducer HBM U9B / 1 kN (left), Interface $1610 / 2.2 \mathrm{kN}$ (right), all signals low-pass filtered at $20 \mathrm{kHz}$. 

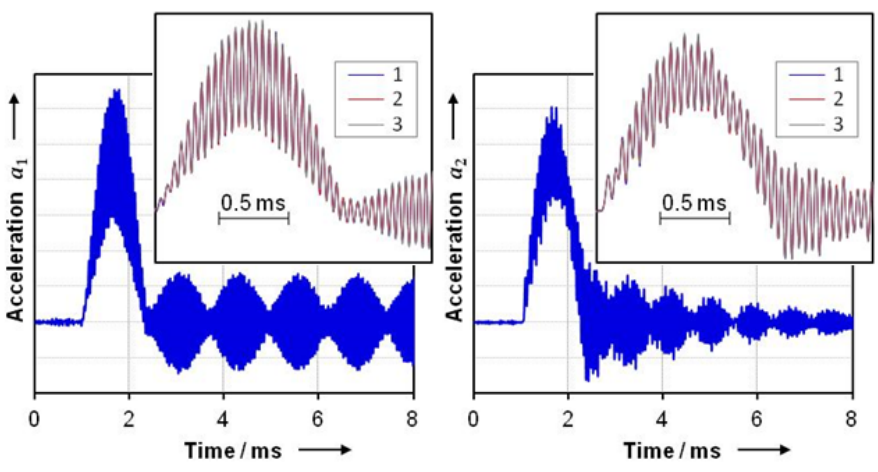

Figure 4. Shock-excited ringing of the acceleration signals:

force transducer HBM U9B / $1 \mathrm{kN}$ with load button of $7.0 \mathrm{~g}$, insets show 3 repetitive measurements (span $2 \mathrm{~ms}$ ), all signals low-pass filtered at $20 \mathrm{kHz}$.

Supplementary measurement data that might be beneficial for the subsequent parameter identification could be obtained by applying an additional load button (see Figure 5). Such a modification of the mechanical impact configuration alters the transducer's dynamic response as the increased mass at the force introduction bolt lowers the transducer's fundamental resonance frequency, which basically is a function of the transducer's head mass and its elastic coupling (see Section 3.1). To achieve well-defined, reproducible testing conditions, the threaded connections of all mechanical couplings were fastened with a defined torque in each case.

Typical shock measurements obtained with two different transducers (HBM U9B / $1 \mathrm{kN}$, Interface $1610 / 2.2 \mathrm{kN}$ ) are presented as examples in the following paragraphs. The plots show the three acquired measurement signals, which are the accelerations $a_{1}$ and $a_{2}$ of both mass bodies derived by differentiation of the vibrometer signals, as well as the transducer output signal force $F$.

The first example in Figure 4 obtained with the HBM U9B / $1 \mathrm{kN}$ demonstrates that the acceleration signals can experience strong shock-excited noise which probably originates from modal oscillations of the cube-shaped mass bodies. These high-frequency signal components might have to be filtered correctly for the subsequent parameter identification process. Using a low-pass filter of $20 \mathrm{kHz}$ cut-off frequency, which is already below the expected fundamental resonance of this transducer, the noise is still very strong. The acceleration $a_{1}$ of the impacting mass body exhibits an almost undamped vibration with a superposed beat signal. Similar behaviour is shown by the signal $a_{2}$ of the reacting mass body with the mounted transducer, but the damping is stronger. In general, the repeatability of the observed high-frequency oscillation pattern is excellent, which is demonstrated by three repetitive measurements (Figure 4 insets). The signal responses to excitations of similar shock intensity are nearly indistinguishable
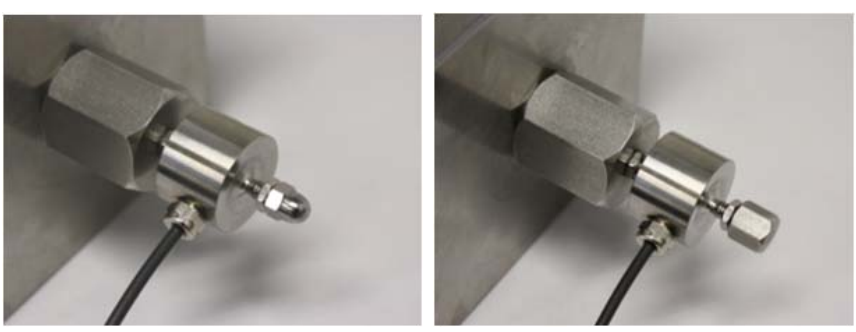

Figure 5. HBM U9B / $1 \mathrm{kN}$ with two load buttons with spherical end faces, increased head mass of $3.1 \mathrm{~g}$ (left) and $7.0 \mathrm{~g}$ (right).

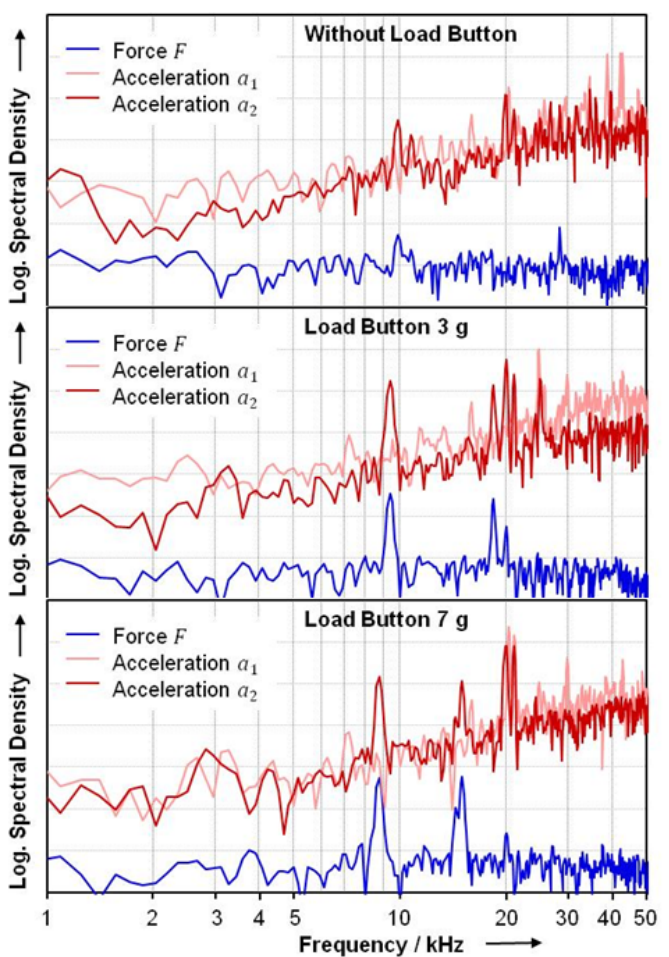

Figure 6. Spectral analysis of the signal ringing for the HBM U9B / $1 \mathrm{kN}$ with different load buttons.

in the time domain.

The effect of an additional load button was investigated with the HBM U9B / $1 \mathrm{kN}$ in order to shift the transducer's fundamental resonance frequency below the above mentioned noise components of the experimental set-up. Figure 5 shows the mounted force transducer with two different load buttons fixed at its threaded rod, and Figure 6 illustrates the measured spectral content of the post-impact signal ringing for the respective configurations. Without an additional head mass, the transducer exhibits an oscillation below $30 \mathrm{kHz}$ that probably is its fundamental resonance. This peak apparently shifts towards
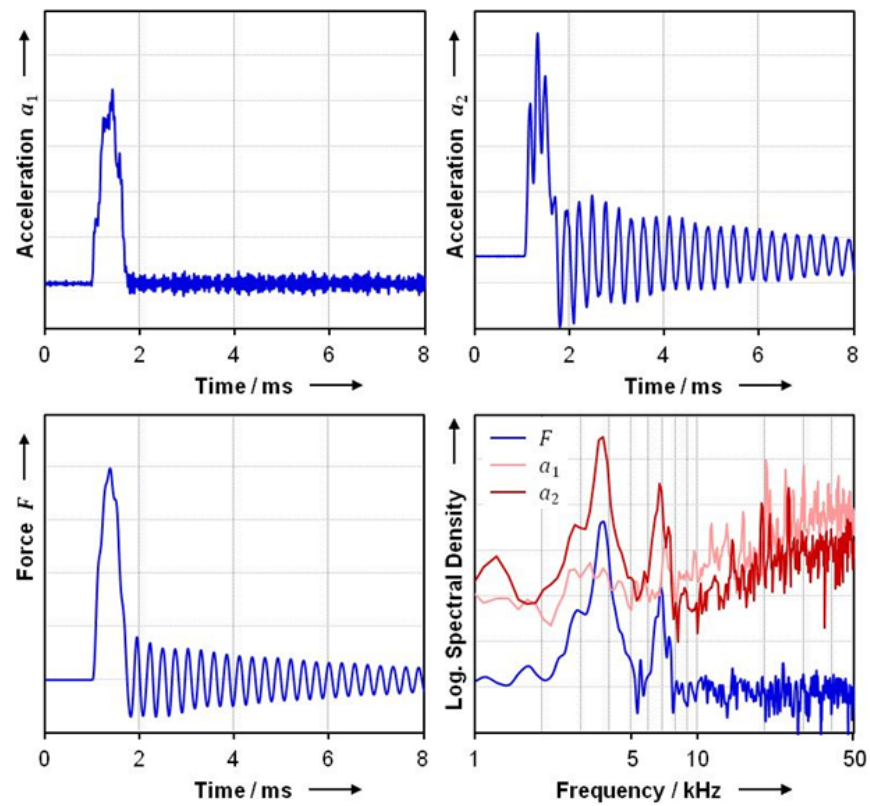

Figure 7. Shock signals in the time domain and frequency domain (signal ringing only) obtained with the force transducer Interface $1610 / 2.2 \mathrm{kN}$, time signals low-pass filtered at $20 \mathrm{kHz}$. 
lower frequencies with the increasing mass of a load button. In addition, a second strong resonance that only slightly depends on the load appears at about $9 \mathrm{kHz}$. This resonance could be identified as a second axial vibration mode due to the mass and elasticity of the transducer housing [7], which is possibly further influenced by a non-rigid mechanical coupling at the base.

The last example in Figure 7 was performed with the Interface $1610 / 2.2 \mathrm{kN}$. Both force $F$ and acceleration $a_{2}$ signals show a strong vibration at $3.7 \mathrm{kHz}$, and the spectral analysis reveals a weaker component at $6.7 \mathrm{kHz}$. With similar amplitudes of the dominant component in both signals, this behaviour actually differs from the previously mentioned tests with the larger transducer model, which may indicate different sources.

Looking at the quite different shock responses obtained with the transducers presented as examples, the identification of the transducer's dynamic parameters from shock measurements may require various analysis tools to provide satisfying results. To elaborate these mathematical methods and analysis procedures for the parameter determination from the experimental data, hundreds of shock force measurements were performed. The large number of tests will further provide an assessment of the reliability of the proposed parameter identification methods.

The first trials on the parameter identification (see Section 3.3) gave consistent results for the force transducer Interface $1610 / 2.2 \mathrm{kN}$ which responded with strong signal ringing, cf. Figure 3. On the other hand, it showed that the smooth shock pulses measured with the HBM U9B / $1 \mathrm{kN}$ apparently contain insufficient information to be able to identify the parameters that describe the transducer's dynamic behaviour. Tests with additional head mass obtained data better suited for the parameter identification process, but it seems that this transducer is more complex and its model description has to be refined. For this reason, the following sections will focus on the first transducer (Interface $1610 / 2.2 \mathrm{kN}$ ), and a dedicated paper will cover the second one (HBM U9B / $1 \mathrm{kN}$ ) in the near future presenting further research.

\section{MODELLING AND PROCEDURES FOR PARAMETER IDENTIFICATION}

\subsection{Model of the force transducer}

Apart from the various influences of a coupled mechanical environment that have to be taken into account to correctly understand a dynamic measurement, the force transducer itself exhibits a dynamic behaviour which is mainly affected by inertia forces due to the motion of its internal mass structure. To describe this behaviour, the force transducer is mathematically modelled by a spring-mass-damper system (Figure 8) consisting of two concentrated masses (base mass $m_{\mathrm{B}}$, head mass $m_{\mathrm{H}}$ ) which are connected by a linear spring element (stiffness $k$, damping $d$ ). The body motions are described by the linear displacement coordinates $x_{\mathrm{B}}$ and $x_{\mathrm{H}}$, and the forces acting at both sides are denoted by the input force $F_{1}$ and the reaction force $F_{2}$. The output signal $F$ of the force transducer is assumed to be proportional to the elongation $x_{\mathrm{H}}-x_{\mathrm{B}}$ of the measuring spring.

Such a mechanical system responds with a characteristic resonance when subjected to a dynamic excitation. Considering a transducer rigidly mounted at its base and neglecting damping, the fundamental resonance frequency $f_{\text {Res }}$ given in equation (1) is a function of $m_{\mathrm{H}}$ and $k$.

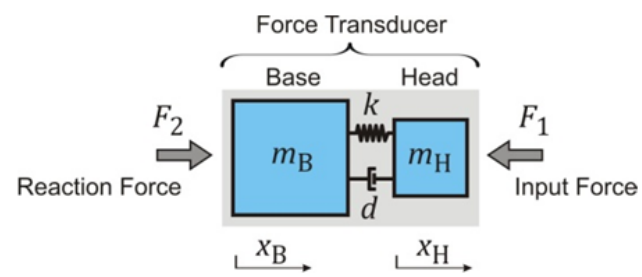

Figure 8. Basic model of a force transducer.

$f_{\text {Res }}=\frac{1}{2 \pi} \sqrt{k / m_{\mathrm{H}}}$

This parameter is often used to judge the dynamic suitability of a force transducer. It is mentioned in the German Directive VDI/VDE 2638 [8] and values are specified in some force transducer data sheets. However, to understand the dynamic measurement behaviour in an application, this single parameter is not sufficient, but rather the four model parameters $m_{\mathrm{B}}, m_{\mathrm{H}}$, $k$ and $d$ have to be known.

\subsection{Model of the calibration device}

By expanding the basic model of the force transducer, the mechanical system of the calibration device with a mounted force transducer is modelled by a one-dimensional multi-body system, which consists of a linear series arrangement of lumped masses coupled by visco-elastic springs.

Models of different degrees of freedom using 3, 4 and 5 model masses were investigated (Figure 9) in order to account for a possible non-rigid coupling at the transducer base as previous shock tests have shown that this mechanical coupling may significantly influence the dynamic measurement behaviour. The three models basically differ in their description of the mechanical adaptation of the transducer to the reacting mass body. The 3-mass model assumes a rigid fixation, whereas the models with 4 and 5 masses describe an elastic coupling. For the 4-mass model, the adapter mass $m_{\mathrm{A}}$ is split and allocated to the neighbouring masses depending on the specific mechanical set-up. In each case, the coupling of an optional load button $m_{\mathrm{L}}$ is assumed to be rigid.

The assumption of a rigid coupling of $m_{\mathrm{L}}$ has to be revised
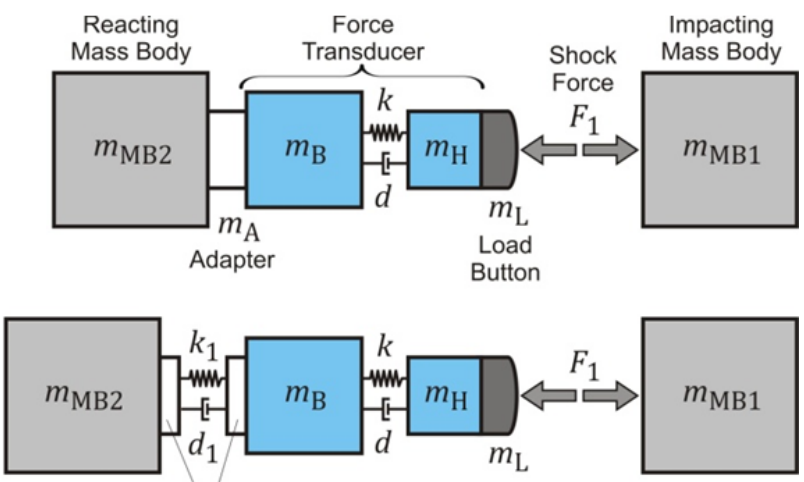

$m_{\mathrm{A}}$

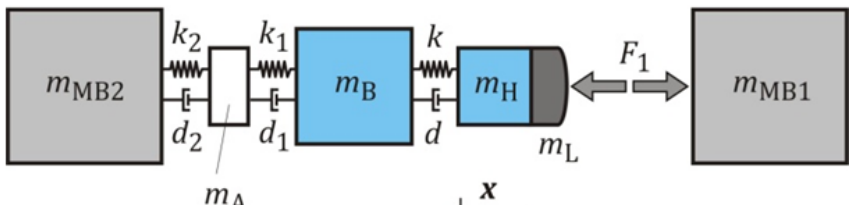

Figure 9. Models of the shock force calibration device: 3-mass model (top), 4-mass model (middle), 5-mass model (bottom). 
if the resulting coupling resonance between $m_{\mathrm{H}}$ and $m_{\mathrm{L}}$ becomes important. This is the case for a considerably large mass $m_{\mathrm{L}}$ and/or a small coupling stiffness which will result in a low resonance frequency that cannot be further neglected. Dynamic force calibrations with sine excitations [9] typically apply large load masses $\left(=m_{\mathrm{L}}\right)$ and the corresponding model descriptions consider an elastic coupling, i.e. a spring element is introduced between $m_{\mathrm{H}}$ and $m_{\mathrm{L}}$. However, the model description of the shock force calibration device may have to be modified accordingly if necessary.

The dynamic behaviour of the model components is expressed by a system of linear ordinary differential equations (ODE) derived from the equilibrium of forces at each mass element. The ODE system has the form

$M \ddot{x}+D \dot{x}+K x=L$

where $\boldsymbol{x}, \dot{\boldsymbol{x}}$ and $\ddot{\boldsymbol{x}}$ are the motion vectors (displacement, velocity, acceleration), and $\boldsymbol{M}, \boldsymbol{D}$, and $\boldsymbol{K}$ denote the matrices for mass, damping and stiffness, and $\boldsymbol{L}$ is the load vector. The mass matrix is diagonal while the damping and stiffness matrices are tri-diagonal with non-zero elements on the main diagonal and the upper and lower sub-diagonal. As an example, the components of the 5-mass model are given in the following equations.

$\boldsymbol{M}=\left[\begin{array}{cccc}m_{\mathrm{H}}+m_{\mathrm{L}} & 0 & 0 & 0 \\ 0 & m_{\mathrm{B}} & 0 & 0 \\ 0 & 0 & m_{\mathrm{A}} & 0 \\ 0 & 0 & 0 & m_{\mathrm{MB} 2}\end{array}\right]$

$\boldsymbol{K}=\left[\begin{array}{cccc}k & -k & 0 & 0 \\ -k & k+k_{1} & -k_{1} & 0 \\ 0 & -k_{1} & k_{1}+k_{2} & -k_{2} \\ 0 & 0 & -k_{2} & k_{2}\end{array}\right]$

$\boldsymbol{D}=\left[\begin{array}{cccc}d & -d & 0 & 0 \\ -d & d+d_{1} & -d_{1} & 0 \\ 0 & -d_{1} & d_{1}+d_{2} & -d_{2} \\ 0 & 0 & -d_{2} & d_{2}\end{array}\right]$

$\boldsymbol{x}=\left[x_{\mathrm{H}}, x_{\mathrm{B}}, x_{\mathrm{A}}, x_{\mathrm{MB} 2}\right]^{\mathrm{T}}$

$\boldsymbol{L}=\left[F_{1}, 0,0,0\right]^{\mathrm{T}}$

The ODE system of the 5-mass model consists of only four equations, as the shock contact, i.e. the coupling between the impacting mass $m_{\mathrm{MB} 1}$ and the force transducer (model mass $\left.m_{\mathrm{H}}+m_{\mathrm{L}}\right)$ is not expressed by another elastic coupling element but rather by the measured shock force $F_{1}$.

Differing from the basic model of the force transducer (Figure 8), the models of the shock calibration device substitute the reaction force $F_{2}$ with the corresponding inertia force calculated by the ODE system, where the motion $x_{\mathrm{MB} 2}$ of the reaction mass body MB2 denotes the input quantity.

Some of the model parameters, such as the mass values of the two mass bodies and the adaptation parts, can be measured before the actual calibration. The remaining parameters, in particular those of the force transducer, have to be inferred from the measured dynamic calibration data or from CAD data.

\subsection{Parameter identification}

Measurement data gained from the calibration experiment are the displacements $x_{\mathrm{MB} 1}$ and $x_{\mathrm{MB} 2}$ of both mass bodies and the corresponding force transducer output signal $F$. Using numerical differentiation by means of finite differences together with low-pass filtering for noise attenuation, the acceleration data $\left(a_{1}, a_{2}\right)$ of the mass bodies is calculated. The measured acceleration $a_{1}$ of the impacting mass body is then used to calculate the system input force $F_{1}$. In order to account for the system change during the measurement interval due to the transient shock contact, we employed a time window to suppress post-impact signal components. For the fit, the transducer output signal $F$ and the acceleration of the reacting mass body $a_{2}$ at the time instants $t_{0}, \ldots, t_{N}$ are considered:

$\boldsymbol{y}\left(t_{n}\right)=\left[F\left(t_{n}\right), a_{2}\left(t_{n}\right)\right]^{\mathrm{T}}$ with $n=0 \ldots N$

Assuming normally distributed measurement noise, we carry out a maximum-likelihood estimation of all parameters by means of non-linear least squares in the time domain. Therefore, a numerical integration method for the differential equations (2) is employed together with the Nelder-Mead nonlinear simplex method for optimization $[10,11]$. The benefit of this optimization method is that it does not require the calculation of derivatives.

The optimization merit function is

$G(\boldsymbol{\theta})=\left\|\boldsymbol{y}-S\left(\boldsymbol{x}\left(t_{0}\right), \boldsymbol{\theta}, F_{1}\right)\right\|^{2}$

where $\|\cdot\|$ is the Euclidean norm, $\boldsymbol{\theta}$ the vector of the sought stiffness and damping parameters, $\boldsymbol{y}$ the measured data and $\boldsymbol{x}\left(t_{0}\right)$ the ODE initial values. In this approach, the mass values of all model components are assumed to be known. The design function $S(\cdot)$ denotes the numerical integration of the ODE (2) corresponding to the considered system model and the calculation of the resulting system output data. For the 5-mass model $S(\cdot)$ is calculated as

$S\left(\boldsymbol{x}\left(t_{0}\right), \boldsymbol{\theta}, F_{1}\right)=\left[\begin{array}{c}k\left(x_{\mathrm{H}}-x_{\mathrm{B}}\right) \\ -\left(k_{2}\left(x_{\mathrm{MB} 2}-\mathrm{x}_{\mathrm{A}}\right)+d_{2}\left(\dot{x}_{\mathrm{MB} 2}-\dot{x}_{\mathrm{A}}\right)\right) / m_{\mathrm{MB} 2}\end{array}\right]$

with the two ODE trajectories denoting the force signal $F$ as a function of the elongation of the measuring spring, and the acceleration $\ddot{x}_{\mathrm{MB} 2}$ of the reacting mass body MB2 calculated from (2).

As the ODE integration is part of the evaluation of the function (10), the calculation of derivatives is a cumbersome analytical and numerical task. Very high precision in the ODE integration would be required to obtain sufficiently precise finite differences for a derivative-based numerical optimization. For this reason, the derivative-free Nelder-Mead simplex method was applied for parameter estimation.

Results of the parameter identification for the transducer Interface $1610 / 2.2 \mathrm{kN}$ are presented in the following. The attenuation of noise in the calculation of acceleration from displacement data was carried out by a 4th order Butterworth low-pass filter of $12 \mathrm{kHz}$ cut-off frequency. Using the model parameters estimated by each model, Figure 10 displays the calculated trajectories of the two elements (force $F$, acceleration $\ddot{x}_{\mathrm{MB} 2}$ ) of the function $S(\cdot)$ and compares them with the corresponding measurement data. For more clarity, the fit residuals are additionally plotted. Regarding the 4-mass model, the adapter mass was fully allocated to the reacting mass body as the adapter is more rigidly coupled to the reacting mass than to the transducer comparing the different mounting conditions (contact area, thread size, mounting torque).

It is seen that the 3 -mass model yields considerably large deviations, in particular in acceleration $a_{2}$. On the other hand, the 4-mass and 5-mass models both show much smaller deviations which demonstrates that the coupling of the 

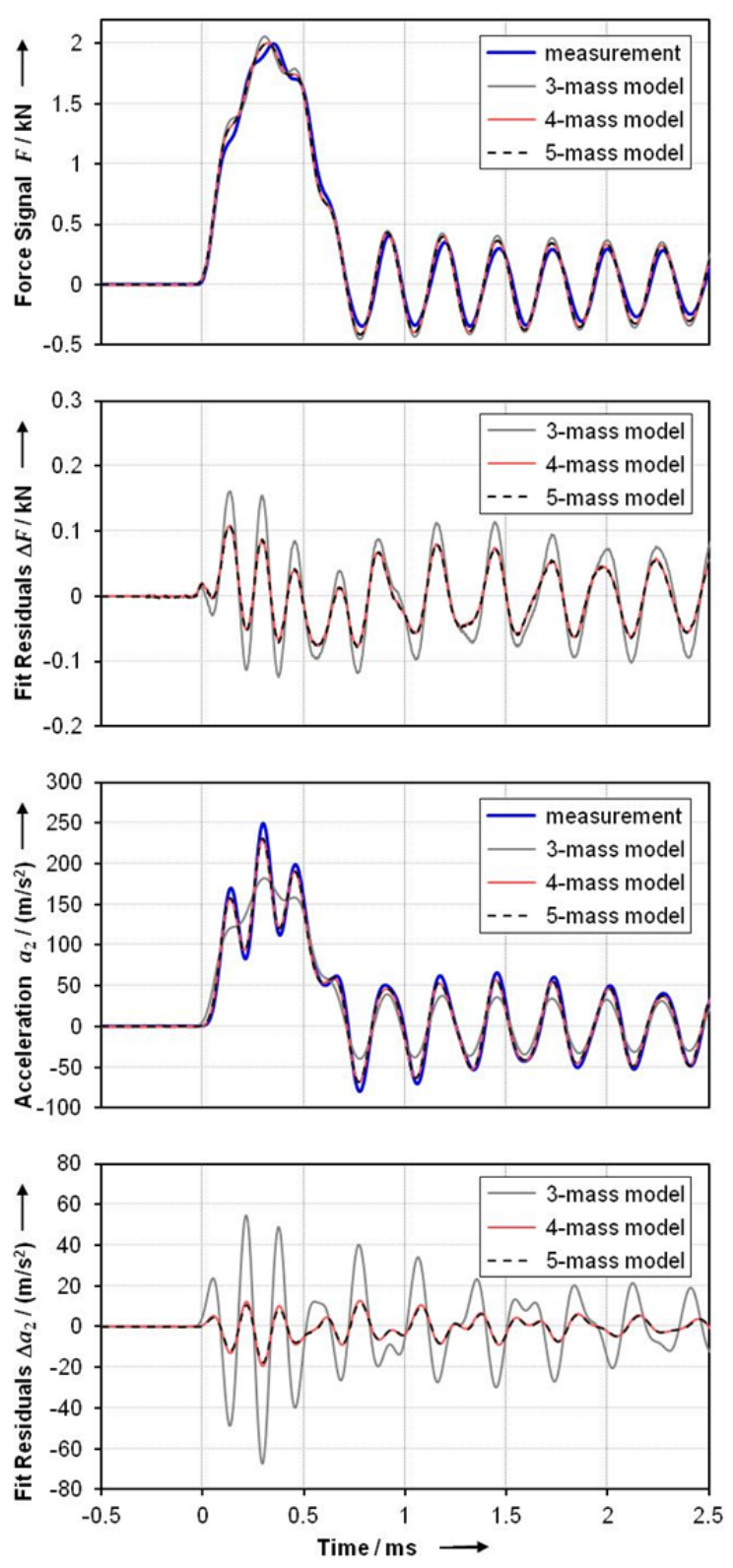

Figure 10. Comparison of modelled and measured shock response signals for the Interface $1610 / 2.2 \mathrm{kN}$ : force signal $F$ (top) and corresponding fit residuals $\Delta F$, acceleration $a_{2}$ of the reacting mass body and corresponding fit residuals $\Delta a_{2}$ (bottom).

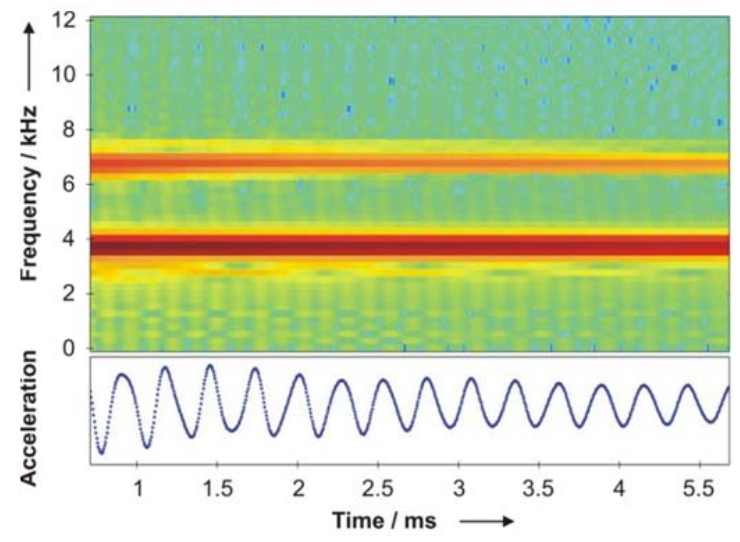

Figure 11. Short-time DFT of the ringing in the measured acceleration $a_{2}$, shock calibration of the Interface $1610 / 2.2 \mathrm{kN}$, the diagram displays the colour-coded acceleration amplitude over time. transducer to the reacting mass body cannot be assumed to be completely rigid for the considered mechanical set-up. As both models achieve a nearly identical fit quality, the chosen 4mass model suffices to describe the system's behaviour. According to Figure 7, there are just two components in the frequency range of interest, which corresponds to such a model. This behaviour is further proved by a short-time DFT analysis of the measured ringing in acceleration $a_{2}$ after the main pulse (Figure 11).

Table 1 summarizes the identified model parameters for the particular data set (as in Figures 10, 11) using models with 3, 4, and 5 masses. For neglected damping, the corresponding resonance frequencies (see Table 2) are calculated from the eigenvalues of the characteristic system matrix of (2) as

$$
\boldsymbol{f}_{\text {Res }}=\frac{1}{2 \pi} \sqrt{\operatorname{eig}\left(\boldsymbol{M}^{-1} \boldsymbol{K}\right)}
$$

It is worth noting that the stiffness of the coupling is ten times greater than that of the transducer's measuring spring. All three models reproduce the lowest resonance at about $3.7 \mathrm{kHz}$ well.

Table 1. Identified model parameters of the Interface $1610 / 2.2 \mathrm{kN}$ using different models, analysis of one shock pulse.

\begin{tabular}{ccrrr}
\hline Parameter & Unit & $\begin{array}{r}\text { 3-Mass } \\
\text { Model }\end{array}$ & $\begin{array}{r}\text { 4-Mass } \\
\text { Model }\end{array}$ & $\begin{array}{c}\text { 5-Mass } \\
\text { Model }\end{array}$ \\
\hline$k$ & $10^{6} \mathrm{~N} / \mathrm{m}$ & 166 & 187 & 186 \\
$d$ & $\mathrm{~kg} / \mathrm{s}$ & 109 & 154 & 154 \\
$k_{1}$ & $10^{6} \mathrm{~N} / \mathrm{m}$ & - & 1743 & 2867 \\
$d_{1}$ & $\mathrm{~kg} / \mathrm{s}$ & - & 539 & 154 \\
$k_{2}$ & $10^{6} \mathrm{~N} / \mathrm{m}$ & - & - & 4680 \\
$d_{2}$ & $\mathrm{~kg} / \mathrm{s}$ & - & - & 1.12 \\
\hline
\end{tabular}

Table 2. Calculated resonance frequencies of the different models.

\begin{tabular}{ccccc}
\hline $\begin{array}{c}\text { Resonant } \\
\text { Frequency }\end{array}$ & Unit & $\begin{array}{c}\text { 3-Mass } \\
\text { Model }\end{array}$ & $\begin{array}{c}\text { 4-Mass } \\
\text { Model }\end{array}$ & $\begin{array}{c}\text { 5-Mass } \\
\text { Model }\end{array}$ \\
\hline$f_{\text {Res }}$ & $\mathrm{kHz}$ & 3.687 & 3.687 & 3.687 \\
$f_{\text {Res,1 }}$ & $\mathrm{kHz}$ & - & 6.695 & 6.694 \\
$f_{\text {Res,2 }}$ & $\mathrm{kHz}$ & - & - & 35.62 \\
\hline
\end{tabular}
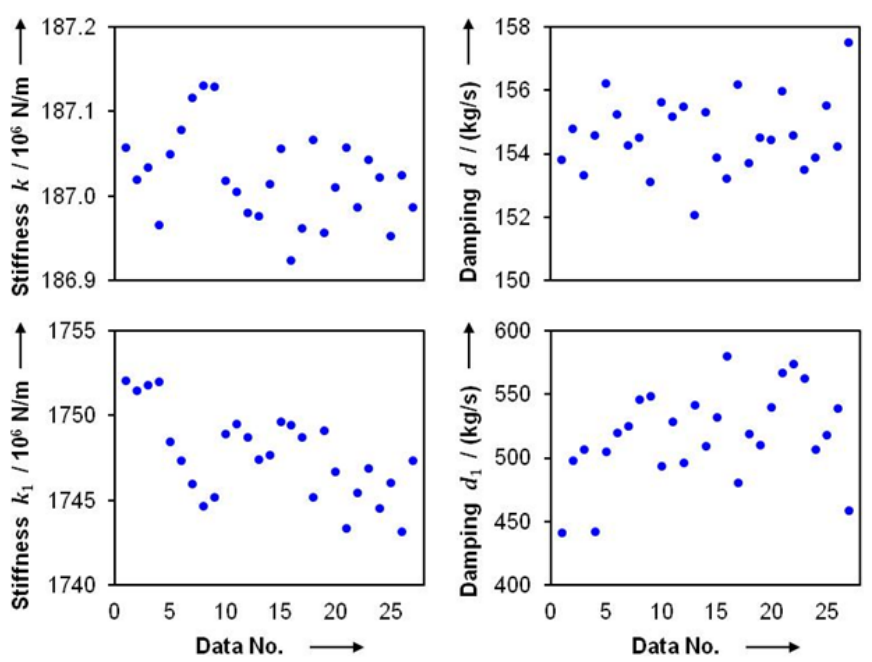

Figure 12. Identified model parameters of the Interface $1610 / 2.2 \mathrm{kN}$ obtained with the 4-mass model, analysis of 27 shock pulses. 
The additional resonance of the 5-mass model falls well outside the considered frequency range, which further confirms that the modelling of the coupling stiffness at both sides of the adapter (cf. Figure 9) does not show any benefit.

Using the 4-mass model, a first analysis of 27 repetitive shock measurements of about $2 \mathrm{kN}$ peak value gives promising results. The identified parameter values of the force transducer Interface $1610 / 2.2 \mathrm{kN}$ (spring stiffness $k$, damping $d$ ) and its coupling $\left(k_{1}, d_{1}\right)$ are plotted in Figure 12.

The relative standard deviations are quite small, in particular those of the stiffness parameters. The larger variance of the damping parameters is probably due to their comparably small influence on the dynamic behaviour of the shock force calibration device, and to the fact that the parameter identification process based on the available measured data is therefore less sensitive.

\section{OUTLOOK}

At the moment it is far too early to specify associated uncertainties for the identified parameters due to numerous factors that may have an influence on the parameter identification process, e.g. the fitting methods, data length, weighting, windowing and filtering. In addition, the remaining deviations observed in the time domain still need some explanation. In any case, the investigated models cannot reproduce the additional spectral peaks at higher frequencies (cf. Figure 7). In the end, all these topics have to be covered in future research.

The parameter identification presented as an example was obtained with shock pulses of considerably strong post-impact signal ringing. Some first trials on the identification of smooth pulses of almost no ringing (cf. Figure 3 left) indicate that the excited modal oscillations presumably carry the crucial information to unambiguously identify the model parameters of a multi-body system having more than one degree of freedom. These findings as well as future investigations on this topic will be presented in a dedicated publication.

The analysis of various measurements with an identical force transducer under modified measurement conditions (e.g. pulse intensities, mechanical adaptations, various mounting conditions) will eventually allow a verification of the suitability of the mechanical model and the data analysis methods applied. In particular, it will demonstrate the influence of various disturbances, e.g. from high-frequency modal oscillations [2] not explained by the chosen model, on the estimation of the model parameters of interest. The result of these investigations will then be incorporated into an extended system model if necessary.

\section{CONCLUSIONS}

This paper presented new research on the model-based dynamic calibration of force transducers by using shock excitations. By analysing experimental shock measurements obtained with force transducers of different structural design, size, weight and mechanical coupling, the suitability of the mathematical models and the applied methods for the estimation of the transducer's model parameters were described and discussed. A first parameter identification applied to shock data obtained with a force transducer that responds with strong shock-excited ringing gave satisfying results.

Future research will investigate the numerous influences on the parameter identification process, as well as the application of the proposed methods to other types of force transducers, e.g. those that respond without strong signal ringing. It will also focus on the comparison of results from sinusoidal excitation experiments and, finally, on the evaluation of the measurement uncertainties for parameter identification.

\section{REFERENCES}

[1] C. Bartoli et al., "Dynamic calibration of force, torque and pressure sensors", Proc. of IMEKO TC3, TC5 and TC22 Int. Conf., Cape Town, South Africa, 2014.

[2] M. Kobusch, L. Klaus, T. Bruns, "Model-based analysis of the dynamic behaviour of a $250 \mathrm{kN}$ shock force calibration device", XX IMEKO World Congress, Busan, 2012, Republic of Korea.

[3] M. Kobusch, A. Link, A. Buss, T. Bruns, "Comparison of shock and sine force calibration methods", Proc. of IMEKO TC3 \& TC16 \& TC22 Int. Conf., Merida, Mexico, 2007.

[4] M. Kobusch, T. Bruns, L. Klaus, M. Müller, "The 250 kN primary shock force calibration device at PTB”, Measurement: 46 (2012), 5, 1757 - 1761.

[5] M. Kobusch, T. Bruns, "Uncertainty contributions of the impact force machine at PTB", Proc. of the XVIII IMEKO World Congress, Rio de Janeiro, Brazil, 2006.

[6] M. Kobusch, "Influence of mounting torque on the stiffness and damping parameters of the dynamic model of a $250 \mathrm{kN}$ shock force calibration device", 7th Workshop on Analysis of Dynamic Measurements, Paris, France, 2012.

[7] M. Kobusch, S. Eichstädt, L. Klaus, T. Bruns, "Analysis of shock force measurements for the model-based dynamic calibration", 8th Workshop on Analysis of Dyn. Meas., Turin, Italy, 2014.

[8] VDI-Richtlinie VDI/VDE/DKD 2638, Kenngrößen für Kraftaufnehmer - Begriffe und Definitionen, Beuth, 2006.

[9] C. Schlegel et al., "Traceable periodic force measurement", Metrologia, vol. 49, 224-235, 2012.

[10] J. Nocedal, S. J. Wright, Numerical Optimization, Springer Series in Operations Research, 1999.

[11] S. Eichstädt, C. Elster, "Reliable uncertainty evaluation for ODE parameter estimation - a comparison”, J. Phys. 490, 1, 2014. 